\title{
On modeling of lifetime data using three-parameter generalized lindley and generalized gamma distributions
}

\begin{abstract}
The analysis and modeling of lifetime data are crucial in almost all applied sciences including behavioral sciences, medicine, insurance, engineering, and finance, amongst others. In this paper an attempt has been made for comparative study of generalized Lindley distribution (GLD) introduced by Zakerzadeh \& Dolati ${ }^{1}$ and generalized gamma distribution (GGD) introduced by $\mathrm{Stacy}^{2}$ for modeling lifetime data from different fields of knowledge. The goodness of fit for both GLD and GGD , based on maximum likelihood estimates, shows that GGD gives much closer fit than GLD in majority of data sets and hence GGD can be considered as an important tool for modeling lifetime data over GLD.
\end{abstract}

Volume 4 Issue 7 - 2016

Rama Shanker, Kamlesh Kumar Shukla

Department of Statistics, Eritrea Institute of Technology, Eritrea

Correspondence: Rama Shanker, Department of Statistics, Eritrea Institute of Technology, Eritrea, Email shankerrama2009@gmail.com

Received: September 19, 2016 | Published: December 09, 2016

Keywords: generalized lindley distribution, generalized gamma distribution, lifetime data, estimation of parameter, goodness of fit

\section{Introduction}

In reliability analysis the time to the occurrence of event of interest is the lifetime or survival time or failure time. The event may be failure of a piece of equipment, death of a person, development (or remission) of symptoms of disease, health code violation (or compliance). The statistical analysis and the modeling of lifetime data are crucial for statisticians and research workers in almost all applied sciences including behavioral sciences, engineering, medical science/ biological science, insurance and finance, amongst others.

A number of lifetime distributions are available for modeling lifetime data in statistics including exponential distribution, gamma distribution, Lindley distribution, Weibull distribution and their generalizations, some amongst others.

In this paper various lifetime data have been considered for modeling using three- parameter generalized Lindley distribution (GLD) and generalized gamma distribution (GGD) because gamma, Lindley and exponential distributions are particular cases of GLD whereas gamma, Weibull and exponential distributions are particular cases of GGD.

\section{Generalized lindley distribution}

The probability density function of three-parameter generalized Lindley distribution (GLD) introduced by Zakerzadeh \& Dolati ${ }^{1}$ having parameters $\alpha, \beta$, and $\theta$ is given by

$$
f_{1}(x ; \alpha, \beta, \theta)=\frac{\theta^{\alpha+1}}{(\beta+\theta)} \frac{x^{\alpha-1}}{\Gamma(\alpha+1)}(\alpha+\beta x) e^{-\theta x} ; x>0, \alpha>0, \beta>0, \theta>0
$$

Clearly the gamma distribution, the Lindley $^{3}$ distribution and the exponential distribution are particular cases of (2.1) for $(\beta=0)$ , $(\alpha=1, \beta=0)$ and $(\alpha=1, \beta=0)$ respectively. The discussion about its properties, estimation of parameters and applications are available in Zakerzadeh \& Dolati. ${ }^{1}$ Ghitany et al., ${ }^{4}$ have detailed study about various properties of Lindley distribution, estimation of parameter and application for modeling waiting time data in a bank. Shanker et al., ${ }^{5}$ have detailed and comparative study about modeling of lifetime data using one parameter Lindley and exponential distributions.
The corresponding distribution function of the GLD can be obtained as

$$
\begin{gathered}
F_{1}(x ; \alpha, \beta, \theta)=1-\frac{\alpha(\beta+\theta) \Gamma(\alpha, \theta x)+\beta(\theta x)^{\alpha} e^{-\theta x}}{(\beta+\theta) \Gamma(\alpha+1)} ; \\
x>0, \alpha>0, \beta>0, \theta>0
\end{gathered}
$$

Where $\Gamma(\alpha, z)$ is the upper incomplete gamma function defined as

$$
(\alpha, z)=\int_{z}^{\infty} e^{-y} y^{\alpha-1} d y ; \alpha>0, z \geq 0
$$

Recently Shanker ${ }^{6}$ has detailed study about GLD and obtained expressions for coefficient of variation, skewness, kurtosis and index of dispersion. Shanker ${ }^{6}$ has also studied its hazard rate function and the mean residual life function.

\section{Generalized gamma distribution}

The probability density function of three-parameter generalized gamma distribution (GGD) introduced by $\mathrm{Stacy}^{2}$ having parameters $\alpha, \beta$, and $\theta$ is given by

$$
f_{2}(x ; \alpha, \beta, \theta)=\frac{\beta \theta^{\alpha}}{\Gamma(\alpha)} x^{\beta \alpha-1} e^{-\theta x^{\beta}} ; x>0, \alpha>0, \beta>0, \theta>0
$$

Where $\alpha$ and $\beta$ are the shape parameter and $\theta$ is the scale parameter. Clearly the gamma distribution, the Weibull distribution and the exponential distribution are particular cases of (3.1) for $(\beta=1),(\alpha=1)$ and $(\alpha=\beta=1)$ respectively. Detailed discussion about GGD is available in Stacy $^{2}$ and parametric estimation for the GGD is available in Stacy \& Mihram. ${ }^{7}$

The cumulative distribution function of the GGD is thus given by

$$
F_{2}(x ; \alpha, \beta, \theta)=1-\frac{\Gamma\left(\alpha, \theta x^{\beta}\right)}{\Gamma(\alpha)}
$$


Where $\Gamma(\alpha, z)$ is the upper incomplete gamma function defined in (2.3)

\section{Maximum likelihood estimation}

Maximum likelihood estimates of the parameters of GLD

Assuming $\left(x_{1}, x_{2}, x_{3}, \ldots, x_{n}\right)$ be a random sample of size $n$ from GLD (2.1), the likelihood function, $L$ of GLD is given by

$L=\left(\frac{\theta^{\alpha+1}}{\beta+\theta}\right)^{n} \frac{1}{(\Gamma(\alpha+1))^{n}} \prod_{i=1}^{n} x_{i}^{\alpha-1}\left(\alpha+\beta x_{i}\right) e^{-n \theta \bar{x}} ; \bar{x}$ being the

sample mean

The natural $\log$ likelihood function is thus obtained as

The maximum likelihood estimate (MLE) $\hat{\theta}, \hat{\alpha}, \hat{\beta}$ of parameters $\theta, \alpha, \beta$ of GLD can be obtained by solving the natural log likelihood equation using R software (Package Stat 4 ).

\section{Maximum likelihood estimates of the parameters of GGD}

Let $\left(x_{1}, x_{2}, x_{3}, \ldots, x_{n}\right)$ be a random sample of size $n$ from GGD (3.1). Then

$$
f\left(x_{i}\right)=\frac{\beta \theta^{\alpha}}{\Gamma(\alpha)}\left(x_{i}\right)^{\beta \alpha-1} e^{-\theta\left(x_{i}\right)^{\beta}}
$$

This gives

$$
\ln f\left(x_{i}\right)=\ln \beta+\alpha \ln \theta-\ln (\Gamma(\alpha))+(\beta \alpha-1) \ln \left(x_{i}\right)-\theta\left(x_{i}\right)^{\beta}
$$

Thus the natural $\log$ likelihood function of the GGD is given by

$$
\ln L=n[\ln \beta+\alpha \ln \theta-\ln (\Gamma(\alpha))]+(\beta \alpha-1) \sum_{i=1}^{n} \ln \left(x_{i}\right)-\theta \sum_{i=1}^{n} x_{i}^{\beta}
$$

The maximum likelihood estimate (MLE) $\hat{\theta}, \hat{\alpha}, \hat{\beta}$ of parameters $\theta, \alpha, \beta$ of GGD can be obtained by solving the natural log likelihood equation using R software (Package Stat 4).

\section{Goodness of fit and applications}

In this section, the goodness of fit and applications of GLD and GGD have been discussed for several lifetime data In order to compare GLD and GGD, $-2 \ln L$ and K-S Statistics KolmogorovSmirnov Statistics) for eighteen data sets have been computed and presented in Table 1.

The formula for K-S Statistics is defined as follow:

$K-S=\operatorname{Sup}\left|F_{n}(x)-F_{0}(x)\right|, \quad$ where $\quad F_{n}(x)$ is the empirical distribution function. The best distribution corresponds to lower values of $-2 \ln L$ and K-S statistics and higher p-values. It is clear from the goodness of fit of GLD and GGD that in most of the data sets except Data sets (1-18) GGD gives much closer fit than GLD for modeling lifetime data.

\section{Conclusion}

The modeling and analysis of lifetime data using lifetime distributions are crucial in almost all applied sciences including behavioral sciences, medicine, insurance, engineering, and finance, amongst others. In this paper an attempt has been made to have a comparative study on modeling of lifetime data on eighteen data sets using three parameters generalized Lindley distribution (GLD) introduced by Zakerzadeh \& Dolati ${ }^{1}$ and generalized gamma distribution (GGD) introduced by Stacy. ${ }^{2}$ Maximum likelihood estimates have been used for fitting both GLD and GGD. The goodness of fit for both GLD and GGD shows that GGD gives much closer fit than GLD in majority of data sets and hence GGD can be considered as an important tool for modeling lifetime data over GLD.

Data Set I The data set represents the strength of $1.5 \mathrm{~cm}$ glass fibers measured at the National Physical Laboratory, England. Unfortunately, the units of measurements are not given in the paper, and they are taken from Smith \& Naylor ${ }^{8}$

\begin{tabular}{lllllllllllll}
\hline 0.55 & 0.93 & 1.25 & 1.36 & 1.49 & 1.52 & 1.58 & 1.61 & 1.64 & 1.68 & 1.73 & 1.81 & 1.76 \\
2.00 & 0.74 & 1.04 & 1.27 & 1.39 & 1.49 & 1.53 & 1.59 & 1.61 & 1.66 & 1.68 & 1.76 & 1.69 \\
1.82 & 2.01 & 0.77 & 1.11 & 1.28 & 1.42 & 1.50 & 1.54 & 1.60 & 1.62 & 1.66 & 1.69 & \\
1.76 & 1.84 & 2.24 & 0.81 & 1.13 & 1.29 & 1.48 & 1.51 & 1.55 & 1.61 & 1.62 & 1.66 & 1.65 \\
1.70 & 1.77 & 1.84 & 0.84 & 1.24 & 1.30 & 1.48 & 1.51 & 1.55 & 1.61 & 1.63 & 1.67 & \\
1.70 & 1.78 & 1.89 & & & & & & & & & &
\end{tabular}

Data Set 2 The data is given by Birnbaum \& Saunders 9 on the fatigue life of $606 \mathrm{I}-\mathrm{T} 6$ aluminum coupons cut parallel to the direction of rolling and oscillated

\begin{tabular}{|c|c|c|c|c|c|c|c|c|c|c|c|}
\hline 5 & 25 & 31 & 32 & 34 & 35 & 38 & 39 & 39 & 40 & 42 & 43 \\
\hline 43 & 43 & 44 & 44 & 47 & 47 & 48 & 49 & 49 & 49 & 51 & 54 \\
\hline 55 & 55 & 55 & 56 & 56 & 56 & 58 & 59 & 59 & 59 & 59 & 59 \\
\hline 63 & 63 & 64 & 64 & 65 & 65 & 65 & 66 & 66 & 66 & 66 & 66 \\
\hline 67 & 67 & 67 & 68 & 69 & 69 & 69 & 69 & 71 & 71 & 72 & 73 \\
\hline 73 & 73 & 74 & 74 & 76 & 76 & 77 & 77 & 77 & 77 & 77 & 77 \\
\hline 79 & 79 & 80 & 81 & 83 & 84 & 84 & 86 & 86 & 87 & 90 & 91 \\
\hline 92 & 92 & 92 & 92 & 93 & 94 & 97 & 98 & 98 & 99 & 101 & 103 \\
\hline 105 & 109 & 136 & 147 & & & & & & & & \\
\hline
\end{tabular}
at 18 cycles per second. The data set consists of 101 observations with maximum stress per cycle 31,000 psi. The data $\left(\times 10^{-3}\right)$ are presented below (after subtracting 65) 
Data Set 3 The data set is from Lawless. ${ }^{10}$ The data given arose in tests on endurance of deep groove ball bearings. The data are the number of million revolutions before failure for each of the 23 ball bearings in the life tests and they are

\begin{tabular}{llllllllllll}
17.88 & 28.92 & 33.00 & 41.52 & 42.12 & 45.60 & 48.80 & 51.84 & 51.96 & 54.12 & 55.56 & 67.80 \\
68.44 & 68.64 & 68.88 & 84.12 & 93.12 & 98.64 & 105.12 & 105.84 & 127.92 & 128.04 & 173.40 & \\
\hline
\end{tabular}

Data Set 4 The data is from Picciotto" and arose in test on the cycle at which the Yarn failed. The data are the number of cycles until failure of the yarn and they are

\begin{tabular}{|c|c|c|c|c|c|c|c|c|c|c|c|}
\hline 86 & 146 & 251 & 653 & 98 & 249 & 400 & 292 & $13 \mid$ & 169 & 175 & 176 \\
\hline 76 & 264 & 15 & 364 & 195 & 262 & 88 & 264 & 157 & 220 & 42 & 321 \\
\hline 180 & 198 & 38 & 20 & 61 & $|2|$ & 282 & 224 & 149 & 180 & 325 & 250 \\
\hline 196 & 90 & 229 & 166 & 38 & 337 & 65 & $|5|$ & $34 I$ & 40 & 40 & 135 \\
\hline 597 & 246 & 211 & 180 & 93 & 315 & 353 & 571 & 124 & 279 & 81 & 186 \\
\hline 497 & 182 & 423 & 185 & 229 & 400 & 338 & 290 & 398 & 7I & 246 & 185 \\
\hline 188 & 568 & 55 & 55 & 61 & 244 & 20 & 284 & 393 & 396 & 203 & 829 \\
\hline 239 & 236 & 286 & 194 & 277 & 143 & 198 & 264 & 105 & 203 & 124 & 137 \\
\hline 135 & 350 & 193 & 188 & & & & & & & & \\
\hline
\end{tabular}

Data Set $\mathbf{5}$ This data represents the survival times (in days) of 72 guinea pigs infected with virulent tubercle bacilli, observed and reported by Bjerkedal'

\begin{tabular}{|c|c|c|c|c|c|c|c|c|c|c|c|}
\hline 10 & 33 & 44 & 56 & 59 & 72 & 74 & 77 & 92 & 93 & 96 & 100 \\
\hline 100 & 102 & 105 & 107 & 107 & 108 & 108 & 108 & 109 & 112 & 113 & 115 \\
\hline 116 & 120 & 121 & 122 & 122 & 124 & 130 & 134 & 136 & 139 & 144 & 146 \\
\hline 153 & 159 & 160 & 163 & 163 & 168 & $|7|$ & 172 & 176 & 183 & 195 & 196 \\
\hline 197 & 202 & 213 & 215 & 216 & 222 & 230 & 231 & 240 & 245 & 251 & 253 \\
\hline 254 & 254 & 278 & 293 & 327 & 342 & 347 & 361 & 402 & 432 & 458 & 555 \\
\hline
\end{tabular}

Data Set 6 This data is related with behavioral sciences, collected by Balakrishnan N et al. [13]:The scale "General Rating of Affective Symptoms for Preschoolers (GRASP)" measures behavioral and emotional problems of children, which can be classified with depressive condition or not according to this scale. A study conducted by the authors in a city located at the south part of Chile has allowed collecting real data corresponding to the scores of the GRASP scale of children with frequency in parenthesis, which are

\begin{tabular}{lllllll}
\hline $19(16)$ & $20(15)$ & $21(14)$ & $22(9)$ & $23(12)$ & $24(10)$ & $25(6)$ \\
$26(9)$ & $27(8)$ & $28(5)$ & $29(6)$ & $30(4)$ & $31(3)$ & $32(4)$ \\
33 & 34 & $35(4)$ & $36(2)$ & $37(2)$ & 39 & 42 \\
\hline
\end{tabular}

Data Set 7 The data set reported by Efron ${ }^{14}$ represent the survival times of a group of patients suffering from Head and Neck cancer disease and treated using radiotherapy $(\mathrm{RT})$.

\begin{tabular}{llllllllllll}
\hline 6.53 & 7 & 10.42 & 14.48 & 16.10 & 22.70 & 34 & 41.55 & 42 & 45.28 & 49.40 & 53.62 \\
63 & 64 & 83 & 84 & 91 & 108 & 112 & 129 & 133 & 133 & 139 & 140 \\
140 & 146 & 149 & 154 & 157 & 160 & 160 & 165 & 146 & 149 & 154 & 157 \\
160 & 160 & 165 & 173 & 176 & 218 & 225 & 241 & 248 & 273 & 277 & 297 \\
405 & 417 & 420 & 440 & 523 & 583 & 594 & 1101 & 1146 & 1417 & & \\
\hline
\end{tabular}

Data Set 8 The data set reported by Efron ${ }^{14}$ represent the survival times of a group of patients suffering from Head and Neck cancer disease and treated using a combination of radiotherapy and chemotherapy $(\mathrm{RT}+\mathrm{CT})$

\begin{tabular}{llllllllllll}
\hline 12.20 & 23.56 & 23.74 & 25.87 & 31.98 & 37 & 41.35 & 47.38 & 55.46 & 58.36 & 63.47 & 68.46 \\
78.26 & 74.47 & 81.43 & 84 & 92 & 94 & 110 & 112 & 119 & 127 & 130 & 133 \\
140 & 146 & 155 & 159 & 173 & 179 & 194 & 195 & 209 & 249 & 281 & 319 \\
339 & 432 & 469 & 519 & 633 & 725 & 817 & 1776 & & & \\
\hline
\end{tabular}


Data set 9 This data set represents remission times (in months) of a random sample of 128 bladder cancer patients reported in Lee \& Wang 15

\begin{tabular}{|c|c|c|c|c|c|c|c|c|c|c|c|}
\hline 0.08 & 2.09 & 3.48 & 4.87 & 6.94 & 8.66 & 13.11 & 23.63 & 0.20 & 2.23 & 3.52 & 4.98 \\
\hline 6.97 & 9.02 & 13.29 & 0.40 & 2.26 & 3.57 & 5.06 & 7.09 & 9.22 & 13.80 & 25.74 & 0.50 \\
\hline 2.46 & 3.64 & 5.09 & 7.26 & 9.47 & 14.24 & 25.82 & 0.51 & 2.54 & 3.70 & 5.17 & 7.28 \\
\hline 9.74 & 14.76 & 6.31 & 0.81 & 2.62 & 3.82 & 5.32 & 7.32 & 10.06 & 14.77 & 32.15 & 2.64 \\
\hline 3.88 & 5.32 & 7.39 & 10.34 & 14.83 & 34.26 & 0.90 & 2.69 & 4.18 & 5.34 & 7.59 & 10.66 \\
\hline 15.96 & 36.66 & 1.05 & 2.69 & 4.23 & $5.4 I$ & 7.62 & 10.75 & 16.62 & 43.01 & 1.19 & 2.75 \\
\hline 4.26 & $5.4 I$ & 7.63 & 17.12 & 46.12 & 1.26 & 2.83 & 4.33 & 5.49 & 7.66 & 11.25 & 17.14 \\
\hline 79.05 & 1.35 & 2.87 & 5.62 & 7.87 & 11.64 & 17.36 & 1.40 & 3.02 & 4.34 & 5.71 & 7.93 \\
\hline II.79 & 18.10 & 1.46 & 4.40 & 5.85 & 8.26 & 11.98 & 19.13 & 1.76 & 3.25 & 4.50 & 6.25 \\
\hline 8.37 & 12.02 & 2.02 & 3.31 & 4.51 & 6.54 & 8.53 & 12.03 & 20.28 & 2.02 & 3.36 & 6.76 \\
\hline 12.07 & 21.73 & 2.07 & 3.36 & 6.93 & 8.65 & 12.63 & 22.69 & & & & \\
\hline
\end{tabular}

Data Set 10 This data set is given by Linhart \& Zucchini [16] which represents the failure times of the air conditioning system of an airplane

$\begin{array}{llllllllllllll}23 & 261 & 87 & 7 & 120 & 14 & 62 & 47 & 225 & 71 & 246 & 21 & 14 & 11 \\ 42 & 20 & 5 & 12 & 120 & 11 & 3 & 14 & 71 & 11 & 14 \\ 16 & 901 & 16 & 52 & 95 & & & & & & \end{array}$

Data Set I I This data set used by Bhaumik et al., ${ }^{17}$ is vinyl chloride data obtained from clean up gradient monitoring wells in mg/l

$\begin{array}{lllllllllllll}5.1 & 1.2 & 1.3 & 0.6 & 0.5 & 2.4 & 0.5 & 1.1 & 8 & 0.8 & 0.4 & 0.6 & \\ 0.9 & 0.4 & 2 & 0.5 & 5.3 & 3.2 & 2.7 & 2.9 & 2.5 & 2.3 & 1 \\ 0.1 & 0.1 & 1.8 & 0.9 & 2 & 4 & 6.8 & 1.2 & 0.4 & 0.2 & \end{array}$

Data set 12 This data set represents the waiting times (in minutes) before service of 100 Bank customers and examined and analyzed by Ghitany et al., ${ }^{4}$ for fitting the Lindley ${ }^{3}$ distribution

\begin{tabular}{|c|c|c|c|c|c|c|c|c|c|c|c|}
\hline 0.8 & 0.8 & 1.3 & 1.5 & 1.8 & 1.9 & 1.9 & 2.1 & 2.6 & 2.7 & 2.9 & 3.1 \\
\hline 3.2 & 3.3 & 3.5 & 3.6 & 4 & 4.1 & 4.2 & 4.2 & 4.3 & 4.3 & 4.4 & 4.4 \\
\hline 4.6 & 4.7 & 4.7 & 4.8 & 4.9 & 4.9 & 5 & 5.3 & 5.5 & 5.7 & 5.7 & 6.1 \\
\hline 6.2 & 6.2 & 6.2 & 6.3 & 6.7 & 6.9 & 7.1 & 7.1 & 7.1 & 7.1 & 7.4 & 7.6 \\
\hline 7.7 & 8 & 8.2 & 8.6 & 8.6 & 8.6 & 8.8 & 8.8 & 8.9 & 8.9 & 9.5 & 9.6 \\
\hline 9.7 & 9.8 & 10.7 & 10.9 & 11 & 11 & II.I & 11.2 & 11.2 & II.5 & 11.9 & 12.4 \\
\hline 12.5 & 12.9 & 13 & 13.1 & 13.3 & 13.6 & 13.7 & 13.9 & 14.1 & 15.4 & 15.4 & 17.3 \\
\hline 17.3 & 18.1 & 18.2 & 18.4 & 18.9 & 19 & 19.9 & 20.6 & 21.3 & 21.4 & 21.9 & 23 \\
\hline 27 & 31.6 & 33.1 & 38.5 & & & & & & & & \\
\hline
\end{tabular}

Data Set I 3 This data is for the times between successive failures of air conditioning equipment in a Boeing 720 airplane, Proschan ${ }^{18}$

$\begin{array}{llllllllllll}74 & 57 & 48 & 29 & 502 & 12 & 70 & 21 & 29 & 386 & 59 & \\ 153 & 26 & 326 & & & & & & & & & \end{array}$

Data set I4 This data set represents the lifetime's data relating to relief times (in minutes) of 20 patients receiving an analgesic and reported by Gross \& Clark ${ }^{19}$

$\begin{array}{llllllllllll}\text { I.I } & 1.4 & 1.3 & 1.7 & 1.9 & 1.8 & 1.6 & 2.2 & 1.7 & 2.7 & 4.1 & 1.8 \\ 1.5 & 1.2 & 1.4 & 3 & 1.7 & 2.3 & 1.6 & 2 & & & & \end{array}$

Data Set I 5 This data set is the strength data of glass of the aircraft window reported by Fuller et al., ${ }^{20}$

\begin{tabular}{llllllllllll}
\hline 18.83 & 20.8 & 21.657 & 23.03 & 23.23 & 24.05 & 24.321 & 25.5 & 25.52 & 25.8 & 26.69 & 26.77 \\
26.78 & 27.05 & 27.67 & 29.9 & 31.11 & 33.2 & 33.73 & 33.76 & 33.89 & 34.76 & 35.75 & 35.91 \\
36.98 & 37.08 & 37.09 & 39.58 & 44.045 & 45.29 & 45.381 & & & & \\
\hline
\end{tabular}


Data Set 16 The following data represent the tensile strength, measured in GPa, of 69 carbon fibers tested under tension at gauge lengths of $20 \mathrm{~mm}$, Bader \& Priest $^{21}$

\begin{tabular}{lllllllllllll}
\hline 1.312 & 1.314 & 1.479 & 1.552 & 1.700 & 1.803 & 1.861 & 1.865 & 1.944 & 1.958 & 1.966 & 1.997 & 2.95 \\
2.006 & 2.021 & 2.027 & 2.055 & 2.063 & 2.098 & 2.140 & 2.179 & 2.224 & 2.240 & 2.253 & 2.270 & 2.253 \\
2.272 & 2.274 & 2.301 & 2.301 & 2.359 & 2.382 & 2.382 & 2.426 & 2.434 & 2.435 & 2.478 & 2.490 \\
2.511 & 2.514 & 2.535 & 2.554 & 2.566 & 2.570 & 2.586 & 2.629 & 2.633 & 2.642 & 2.648 & 2.684 \\
2.697 & 2.726 & 2.770 & 2.773 & 2.800 & 2.809 & 2.818 & 2.821 & 2.848 & 2.880 & 2.954 & 3.012 \\
3.067 & 3.084 & 3.090 & 3.096 & 3.128 & 3.233 & 3.433 & 3.585 & 3.585 & &
\end{tabular}

Data Set 17 The following data set represents the failure times (in minutes) for a sample of I5 electronic components in an accelerated life test, Lawless. ${ }^{10}$

\begin{tabular}{|c|c|c|c|c|c|c|c|c|c|c|c|}
\hline 1.4 & 5.1 & 6.3 & 10.8 & 12.1 & 18.5 & 19.7 & 22.2 & 23.0 & 30.6 & 37.3 & 46.3 \\
\hline 53.9 & 59.8 & 66.2 & & & & & & & & & \\
\hline
\end{tabular}

Data Set 18 The following data set represents the number of cycles to failure for 25100 -cm specimens of yarn, tested at a particular strain level, Lawless ${ }^{10}$

\begin{tabular}{|c|c|c|c|c|c|c|c|c|c|c|c|}
\hline 15 & 20 & 38 & 42 & 61 & 76 & 86 & 98 & $|2|$ & 146 & 149 & 157 \\
\hline 175 & 176 & 180 & 180 & 198 & 220 & 224 & 251 & 264 & 282 & 321 & 325 \\
\hline
\end{tabular}

Table I ML Estimates, -2In L, K-S Statistics and p-values of the fitted distributions of data sets I to 18

\begin{tabular}{|c|c|c|c|c|c|c|c|}
\hline & \multirow{2}{*}{ Model } & \multicolumn{3}{|c|}{ ML Estimates } & \multirow[b]{2}{*}{$-2 \ln L$} & \multirow{2}{*}{$\begin{array}{l}\text { K-S } \\
\text { Statistic }\end{array}$} & \multirow[b]{2}{*}{ P-Value } \\
\hline & & $\hat{\alpha}$ & $\hat{\beta}$ & $\hat{\theta}$ & & & \\
\hline \multirow{2}{*}{ Data I } & GLD & 17. 1792 & 14. 3378 & II.7653 & 47.784 & 0.809 & 0.000 \\
\hline & GGD & 0.6831 & 7.2644 & 0.0176 & 29.238 & 0.796 & 0.000 \\
\hline \multirow{2}{*}{ Data 2} & GLD & 7.0755 & 0.4492 & 0.1152 & 914.950 & 0.098 & 0.281 \\
\hline & GGD & 4.8293 & I.307I & 0.0188 & 912.437 & 0.087 & 0.429 \\
\hline \multirow{2}{*}{ Data 3} & GLD & 3.0404 & 3.5804 & 0.0557 & 226.060 & 0.123 & 0.833 \\
\hline & GGD & 8.8487 & 0.6605 & 0.5387 & 225.932 & 0.112 & 0.908 \\
\hline \multirow{2}{*}{ Data 4} & GLD & 1.8488 & 0.0245 & 0.0114 & 1249.850 & 0.957 & 0.000 \\
\hline & GGD & 2.5118 & 0.9386 & 0.0159 & 1250.768 & 0.954 & 0.000 \\
\hline \multirow{2}{*}{ Data 5} & GLD & 1.0932 & 5.0688 & 0.0209 & 788.575 & 0.439 & 0.000 \\
\hline & GGD & 26.3684 & 0.2718 & 7.9396 & 781.728 & 0.476 & 0.000 \\
\hline \multirow{2}{*}{ Data 6} & GLD & 17.5655 & 5.1916 & 0.6181 & |47.087 & 0.067 & 0.999 \\
\hline & GGD & 14.9397 & I.1094 & 0.3437 & | 47.092 & 0.068 & 0.999 \\
\hline \multirow{2}{*}{ Data 7} & GLD & 0.0557 & 5.0640 & 0.0047 & 744.975 & 0.169 & 0.072 \\
\hline & GGD & 5.5727 & 0.3912 & 0.7576 & 741.716 & 0.142 & 0.193 \\
\hline \multirow{2}{*}{ Data 8} & GLD & 0.0524 & 5.0750 & 0.0047 & 564.096 & 0.150 & 0.248 \\
\hline & GGD & 27.7234 & 0.1822 & II.2554 & 555.636 & 0.079 & 0.921 \\
\hline \multirow{2}{*}{ Data 9} & GLD & I.I85। & 0.0006 & 0.1287 & 822.169 & 0.877 & 0.000 \\
\hline & GGD & 3.8869 & 0.5139 & I.3883 & 816.852 & 0.873 & 0.000 \\
\hline \multirow{2}{*}{ Data 10} & GLD & 0.8114 & 0.0007 & 0.0144 & 304.348 & 0.948 & 0.000 \\
\hline & GGD & 6.4942 & 0.3080 & 2.1333 & 302.680 & 0.933 & 0.000 \\
\hline \multirow{2}{*}{ Data I I } & GLD & 1.0628 & 0.0006 & 0.5647 & I I 0.826 & 0.936 & 0.000 \\
\hline & GGD & 5.9538 & 0.3802 & 5.2747 & $|09.72|$ & 0.927 & 0.000 \\
\hline
\end{tabular}




\begin{tabular}{|c|c|c|c|c|c|c|c|}
\hline & \multirow{2}{*}{ Model } & \multicolumn{3}{|c|}{ ML Estimates } & \multirow{2}{*}{$-2 \ln L$} & \multirow{2}{*}{$\begin{array}{l}\text { K-S } \\
\text { Statistic }\end{array}$} & \multirow{2}{*}{ P-Value } \\
\hline & & $\hat{\alpha}$ & $\hat{\beta}$ & $\hat{\theta}$ & & & \\
\hline \multirow{2}{*}{ Data I2 } & GLD & 2.0093 & 0.0007 & 0.2038 & 634.600 & 0.043 & 0.994 \\
\hline & GGD & 3.8037 & 0.7017 & 0.8028 & 634.035 & 0.036 & 0.999 \\
\hline \multirow{2}{*}{ Data I3 } & GLD & 0.9427 & 0.0003 & 0.0081 & 173.873 & 0.726 & 0.000 \\
\hline & GGD & 26.6637 & 0.1736 & 12.7036 & 170.488 & 0.726 & 0.000 \\
\hline \multirow{2}{*}{ Data I4 } & GLD & 9.6686 & 0.0029 & 5.0891 & 35.637 & 0.609 & 0.000 \\
\hline & GGD & 51.4619 & 0.4350 & 39.4639 & 34.376 & 0.600 & 0.000 \\
\hline \multirow{2}{*}{ Data I5 } & GLD & I7.988I & $|4.61| 1$ & 0.6150 & 208.233 & 0.135 & 0.580 \\
\hline & GGD & 19.672 & 0.9814 & 0.6800 & 208.225 & 0.136 & 0.562 \\
\hline \multirow{2}{*}{ Data 16} & GLD & 22.7198 & 4.7710 & 9.3907 & 101.959 & 0.056 & 0.979 \\
\hline & GGD & 3.5861 & 2.6483 & 0.3044 & 100.581 & 0.044 & 0.999 \\
\hline \multirow{2}{*}{ Data 17} & GLD & 1.2025 & 0.0832 & $0.064 I$ & $128.16 \mid$ & 0.095 & 0.997 \\
\hline & GGD & 0.8597 & 1.4152 & 0.0068 & $|27.93|$ & 0.095 & 0.997 \\
\hline \multirow{2}{*}{ Data 18} & GLD & 0.8186 & 3.9740 & 0.0101 & 304.883 & 0.132 & 0.769 \\
\hline & GGD & 1.9916 & 0.9426 & 0.0152 & 304.928 & 0.139 & 0.719 \\
\hline
\end{tabular}

\section{Acknowledgement}

None.

\section{Conflict of interest}

None.

\section{References}

1. Zakerzadeh H, Dolati A. Generalized Lindley distribution. Journal of Mathematical extension. 2009;3(2):13-25.

2. Stacy EW. A generalization of the gamma distribution. Annals of Mathematical Statistical. 1962;33:1187-1192.

3. Lindley DV. Fiducial distributions and Bayes' Theorem. Journal of the Royal Statistical Society Series B. 1958;20(1):102-107.

4. Ghitany ME, Atieh B, Nadarajah S. Lindley distribution and its Application. Mathematics Computing and Simulation. 2008;78(4):493-506.

5. Shanker R, Hagos F, Sujatha S. On modeling of Lifetimes data using exponential and Lindley distributions. Biometrics \& Biostatistics International Journal. 2015;2(5):1-9.

6. Shanker R. On generalized Lindley distribution and its applications. Communicated; 2016.

7. Stacy EW, Mihram GA. Parametric estimation for a generalized gamma distribution. Technometrics. 1965;7:349-358.

8. Smith RL, Naylor JC. A comparison of Maximum likelihood and Bayesian estimators for the three parameter Weibull distribution. Applied Statistics. 1987;36(3):358-369.

9. Birnbaum ZW, Saunders SC. Estimation for a family of life distributions with applications to fatigue. Journal of Applied Probability. 1969;6(2):328-347.

10. Lawless JF. Statistical models and methods for lifetime data. John Wiley and Sons: New York, USA; 2003.
11. Picciotto R. Tensile fatigue characteristics of a sized polyester/viscose yarn and their effect on weaving performance. Master thesis, North Carolina State: USA; 1970.

12. Bjerkedal T. Acquisition of resistance in guinea pigs infected with different doses of virulent tubercle bacilli. American Journal of Epidemiology. 1960;72(1):130-148.

13. Balakrishnan N, Victor L, Antonio S. A mixture model based on BirnhaumSaunders Distributions. A study conducted by Authors regarding the Scores of the GRASP (General Rating of Affective Symptoms for Preschoolers), in a city located at South Part of the Chile; 2010.

14. Efron B. Logistic regression, survival analysis and the Kaplan-Meier curve. Journal of the American Statistical Association. 1988;83(408):414-425.

15. Lee ET, Wang JW. Statistical methods for survival data analysis. $3^{\text {rd }}$ ed. John Wiley and Sons: New York, USA; 2003.

16. Linhart H, Zucchini W. Model Selection. John Wiley: New York, USA; 1986

17. Bhaumik DK, Kapur K, Gibbons RD. Testing Parameters of a Gamma Distribution for Small Samples. Technometrics. 2009;51(3):326-334.

18. Proschan F. Theoretical explanation of observed decreasing failure rate Technometrics. 1963;5(3):375-383.

19. Gross AJ, Clark VA. Survival Distributions: Reliability Applications in the Biometrical Sciences. John Wiley: New York, USA; 1975.

20. Fuller EJ, Frieman S, Quinn J, et al. Fracture mechanics approach to the design of glass aircraft windows: A case study. SPIE Proc. 1994;2286:419-430.

21. Bader MG, Priest AM. Statistical aspects of fiber and bundle strength in hybrid composites. In: hayashi T, Kawata, K Umekawa S (Eds.), Progressin Science in Engineering Composites: Tokyo, Japan; 1982. $1129-1136 \mathrm{p}$ 